\title{
End-User Perception Towards Pervasive Cardiac Healthcare Services: Benefits, Acceptance, Adoption, Risks, Security, Privacy and Trust.
}

\author{
Anandhi Vivek Dhukaram \& Chris Baber \\ School of Electronic, Electrical \& \\ Computer Engineering \\ University of Birmingham \\ United Kingdom \\ AVD016@bham.ac.uk
}

\author{
Lamia Elloumi \& Bert-Jan van Beijnum \\ $\mathrm{EWI} / \mathrm{BSS}$ \\ Remote monitoring and treatment lab \\ Zuidhorst 113, University of Twente \\ P.O. Box 217, 7500 AE Enschede \\ Netherlands
}

\author{
Paolo De Stefanis \\ LABOR Srl \\ Via G. Peroni 386 c/o Tecnopolo \\ Tiburtino \\ 00131 ROMA \\ Italy
}

\begin{abstract}
This study examined patient and caregiver's perception regarding pervasive healthcare technology using five focus groups and a 31-item questionnaire. To further develop an understanding of the benefits and functionalities that prospective patients deem as either desirable, undesirable, inadequate or in need of further development the study was categorized under 7 main headings: Personal Profile; Benefits; Adoption; Acceptance; Risks; Security, Privacy and Trust; (use of) Cell Phone. This study was completed as part of the European Union BRAVEHEALTH project, aimed at the support of cardiac patients in everyday life using in vivo monitoring and diagnosis, thereby enabling the patient to be more proactive in heath management. Most participants felt that there is a great future for this technology and showed positive response in regards to the potential benefits but are (at present) not willing to adopt the system due to concerns over reliability, like security, privacy and trust.
\end{abstract}

Keywords-pervasive healthcare systems; end user aspects; cardiac patients;

\section{BACKGROUND}

The aging population is creating pressure on healthcare systems in a variety of ways [1]. In 2009, a European Parliament Heart Group identified Cardio-Vascular Disease (CVD) as the major cause of death, killing over 2 million people each year in Europe alone [2]. It was also found that these deaths occur at a younger age in less developed countries, thus impacting on the economic success of these countries. In principle, CVD patients are capable of leading a normal life as long as they are continuously monitored and alerted in the event of an emergency to the emergency services [3]. Pervasive healthcare monitoring systems could shift the paradigm from the traditional event-driven model (i.e., when a specific change in patient condition leads to a medical intervention, such as admission to hospital) to one where patients are continuously monitored for ECG, Pulse Oximetry, blood pressure and other vital signs through the combinations of bio-sensors, smarthome technologies, video-conferencing and various wireless information networks $[4,5,6,7,8,9,10,11,12,13,14,15,16$, 17, 18]. The resulting data could then provide both a form of 'early warning' of changes in patient condition and more tailored advice and guidance to both patients and phyisicians. A wide range of companies, including Philips, Vodafone, Orange, AT\&T Wireless, Avea, and Japan's NTT DoCoMo, Telefonica, Intel, Hewlett Packard is exploring these combinations of technology.

These advancements in pervasive healthcare can not only assist medical monitoring and diagnosis but also help to resolve the social isolation that these patients can face. The use of monitoring systems in the patient's home can provide assistance without limiting or disturbing the patient's daily routine, giving greater comfort, pleasure, and well-being. Thus enabling the patient to be more proactive in heath management, as well as allowing the health care provider to make more informed decisions with real-time data and thus avoiding serious complications [4]

Despite potential benefits, technical maturity of the solutions and the number of pilot applications running across the World, widespread adoption of such solutions in health care delivery are rare. In this paper, we present the concerns and attitudes of cardiac patient's and caregiver's towards the concept of pervasive healthcare systems. The aim is to further develop understanding of the benefits and functionalities that prospective patients deem as either desirable, undesirable, inadequate or in need of further development.

\section{PRIOR RESEARCH}

Pervasive computing is as much about the user as it is about the technology, but it is noteworthy that patients are rarely mentioned as a motivation for the design and development of cardiac healthcare systems. If we aim at introducing 
technologies that will form part of a person's daily life (in much the same as clothing or, to take a more recent analogy, the mobile phone) then it is important to appreciate how potential users of the technologies might consider the impacts, both positive and negative that the technologies will create. There is a growing literature on user perceptions of healthcare systems. Some of the studies that are relevant to this paper have explored issues such as: the relationship between familiarity with mobile devices and ongoing mobile service usage [19]; perceptions, attitudes and concerns of elderly persons towards wireless sensor network technologies [20]; older adults' attitudes towards smart home technologies [21]; consumer sentiment towards RFID healthcare technology [22]; patients' perceptions regarding home telecare [23]. Other studies are focused on one or two values of interest, for example security [24], adoption [25], risks and benefits [26], and comfort and compliance [27].

In a study employing a similar methodology to this paper, Mitzner et al. [30] found a generally positive response from elderly users to homecare technologies, particularly in terms of how the technologies can provide support and convenience for everyday tasks. However, concerns centred on questions of reliability and security. Demiris et al. [21] also indicated that older users appreciated potential benefits of devices and sensors to enhance their healthcare (particularly in terms of detecting problems, calling for help and monitoring physiological data), but raised concerns over the usability of such devices. These studies indicate that older users are (perhaps contrary to popular opinion) positive about the benefits of new technologies, providing that these benefits outweigh the potential negative impacts on their daily lives.

In a study applying a Technology Acceptance Model to healthcare, Winkelman et al. (2005) argue that simply providing technology to patients may have little benefit without also providing a sense of illness ownership (by the patient), a capability to support patient-initiated communication, a level of support that it tailored to the individual patient and mutual trust between patients and medical professionals using the technology. As part of the BRAVEHEALTH project, the question of how best to develop patient-centric healthcare raises each of these questions.

\section{PROPOSED PERVASIVE SYSTEM AND RESEARCH QUESTIONS}

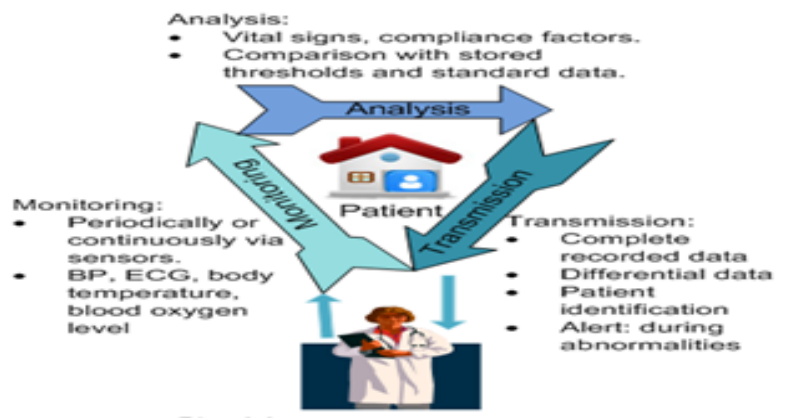

This study is part of BRAVEHEALTH, a European Union funded project. BRAVEHEALTH proposes a patient centric vision to CVD management and treatment, providing people already diagnosed as subjects at risk with a sound solution for continuous and remote monitoring and real time prevention of malignant events as shown in Figure 1.

\section{A. System Architecture}

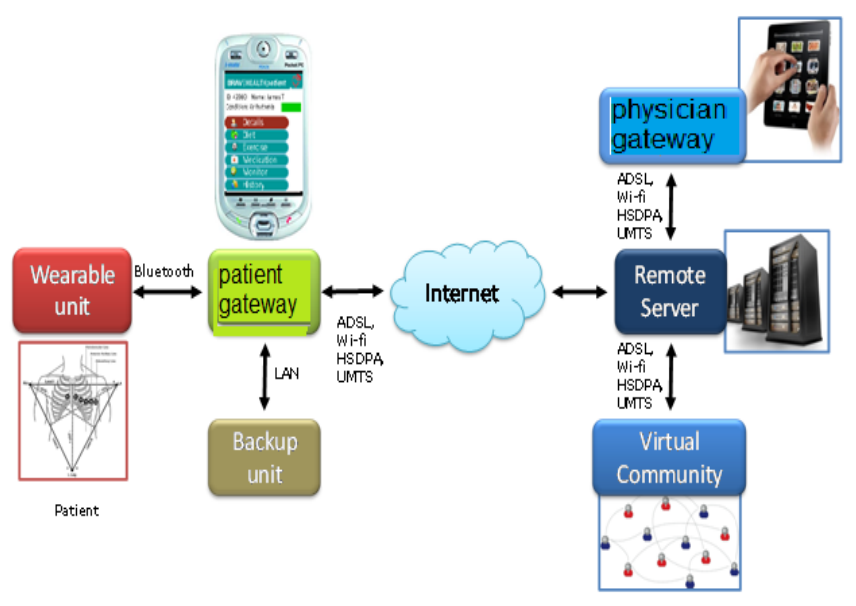

Figure 2: BRAVEHEALTH schematic architecture

Figure 2 shows the preliminary, high-level BRAVEHEALTH system architecture. The aim of such a high level schematic architecture is to provide an appropriate decomposition of the system without delving into the details of interface specification and type information. Separately each of these components itself is a system, and their interaction and combined architecture represents the BRAVEHEALTH system.

\section{B. System Components}

From the technological viewpoint BRAVEHEALTH is based on the devices/platform described below:

1. The Wearable Unit: involves a novel form of embedded processor, integrated into a garment worn by the patient. The unit is able to capture basic physiological data from the patient, together a full Electrocardiogram if required. The data captured by the wearable unit are passed to a Patient Gateway (see below).

2. The Patient Gateway: is typically run on a SmartPhone (current developments involve Windows Mobile and Android platforms). In some instances, the 'gateway' can function as a router, connecting the Wearable Unit to the Server (or other devices), in other instances, the 'gateway' can function as a client, receiving information from the Server to display to the user, and in other instances the 'gateway' can function as a local Server, receiving data from other devices. In most cases, the 'gateway' will be expected to fulfill one or 
more of the functions but that collectively the "patient gateway' supports any combination of these functions.

3. The physician's gateway: provides a means of accessing data collected from the patient. This could be presented on a tablet computer or on the physician's desktop computer. In addition to visualization, the physician's gateway provides a route into the decision support system being developed for the project.

4. The Remote Server: is intended to perform a number of functions, including hosting the BRAVEHEALTH database of patient records. It is central to the Patient Configuration System (include a unique identifier for the patient and clinical information from Electronic Health Records, alerts and notifications), Patient Management System (patient pathology, Risk and notification profile to be managed by the clinicians) and Decision Support System (automatic notifications, physician-patient consultations) capabilities in BRAVEHEALTH. These systems support functionalities on the Wearable Unit, the Patient Gateway and the Physician Gateway.

5. The Mobile Virtual Community: the BRAVEHEALTH Mobile Virtual Communities aim at providing ICT mediated group support to CVD patient with the aim to maintain or improve patients' health status by focusing on relevant disease facets. The disease facets include: diet, physical exercise / activity, medication compliance, diseases information and smoking cessation.

\section{Motivation for this Study}

The basic research questions motivating this study, then, are:

- What are the social and demographic factors associated with these conditions?

- Do patients perceive the benefits of pervasive monitoring systems? What do patients see as the most important benefit?

- Do patients see any risk in using the system or the equipment?

- Do patients use any monitoring equipments at home?

- Do patients currently use the Internet and mobile phones?

- Can patients understand how to use the system?

\section{RESEARCH METHOD}

\section{Focus Groups}

This study adopted focus group interviews [28, 29] and its results for understanding patient's ethical concerns and perceptions towards pervasive healthcare systems. This paper describes the method and results of the study. Broadly, the aim of the study is to generate input for the design of the BRAVEHEALTH system.

Five focus groups were conducted with 34 participants from the West Midlands and Cheshire areas in England. Access to the individuals was supported through the British Heart Foundation and recruitment involved an initial presentation by the researchers and an invitation to attend a small focus group. Consent was obtained from all the participants. The participants were informed that they could leave the session at any time, and all comments were recorded and anonymised prior to analysis. A copy of the initial analysis was sent to each focus group for comment and checking.

Each focus group was given an introduction to BRAVEHEALTH system with scenarios for the patient monitoring system and consultation with physician over the internet or Internet Protocol Television (IPTV) (Figure 1). Each group session consisted of two parts. The first part was focused on patients' everyday decision making. This has been analysed for a separate paper (Dhukaram and Baber, submitted). The second part, to be explored in this paper, focused on patient perception of Telehealth. We were particularly interested in identifying the concerns and attitudes that patients and their caregiver might have of the concept, as well as exploring potential barriers to acceptance, such as variation in computer skills, and technology awareness.

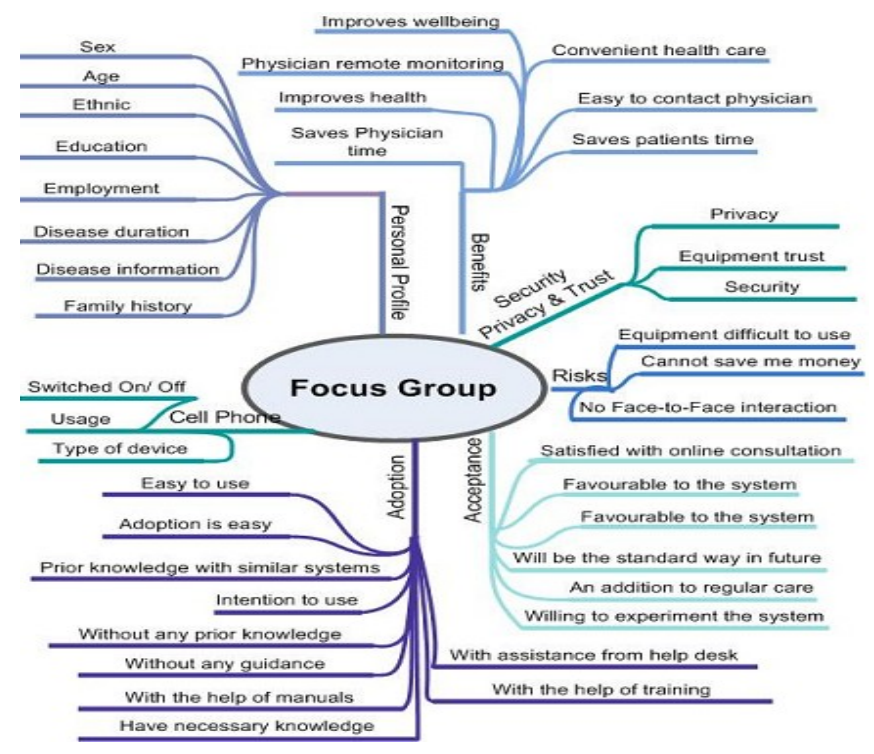

Figure 3: WordCloud of issues raised in Focus Groups

The focus groups resulted in field notes, photographs of participant's mobile phone and transcripts of the interviews. This material was initially collated into a Mindmap [31] (Figure 2) to show key trends in the discussions. These have been categorized under 7 main headings: Personal Profile; Benefits; Security, Privacy and Trust; Adoption; Risks; Acceptance; (use of) Cell Phone. Following this, content analysis was conducted on the transcriptions in terms of these categories. 


\section{FINDINGS}

A questionnaire, designed for the BRAVEHEALTH project, was administered to participants at the end of the focus groups and the results from the questions are summarized in this section.

\section{Personal Profile}

The Study group consisted of 22 Males and 12 Females. While we make no claim for the representativeness of the gender imbalance in this sample, discussion with practitioners suggests that men are more likely to be at risk from cardiac conditions than women and married men more likely to attend support groups (such as those from which the focus groups were drawn). Most of the participants $(29 / 34)$ were in the in the age group of 60 to 79 years of age. The level of education is varied among the study group however most participants (21/ 34) did not attend college. Most of the participants were either not employed (15/34) or retired (14/34).

In terms of history of heart condition, most participants had suffered from the condition for more than 3 years. It is interesting to note that patients were not always able to discover if they had a history of cardiac conditions in their family or had no sought such information.

TABLE 1: DEMOGRAPHICS OF PARTICIPANTS

\begin{tabular}{|l|l|}
\hline & Frequency \\
\hline Gender & \\
Male & 22 \\
Female & 12 \\
\hline Age & \\
$50-59$ & 5 \\
$60-69$ & 18 \\
$70-79$ & 10 \\
$80-89$ & 1 \\
\hline Education & \\
Graduate Education & 5 \\
College & 8 \\
Secondary School & 7 \\
Did obtain qualifications from Secondary & 14 \\
School & \\
\hline Employment & \\
Employed & 5 \\
Not Employed & 15 \\
Retired & 14 \\
\hline History of heart condition: & 4 \\
Less than 6 months & 2 \\
6-1 years & 2 \\
2-3 years & 8 \\
4-5 years & 11 \\
5-10 years & 7 \\
\hline 10+ years & 14 (yes) \\
\hline Have you gathered information from \\
friends and family? & $21(\mathrm{nos})$ \\
\hline Do you have a family history of \\
cardiac problems? & $6(\mathrm{not}$ sure) \\
\hline
\end{tabular}

\section{Benefits}

When asked about the potential benefits of the BRAVEHEALTH concept, the majority of patients (27/34) felt that the technology could save time for both physicians and patients, and allow patients to monitor their condition and improve their well-being. Furthermore, several participants felt that BRAVEHEALTH could be beneficial in improving their general health $(25 / 34)$, or that it could condition $(29 / 34)$. Participants also felt that BRAVEHEALTH is a convenient form of health care delivery for them $(27 / 34)$ and that could make it easier for them to contact the physician (29/34). These findings are illustrated by figure 4 .

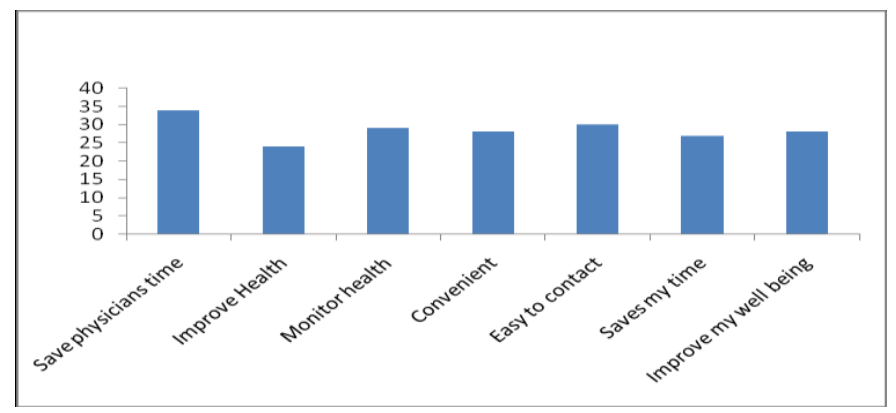

Figure 4: Perceptions of Potential Benefits

Thus, as with the previous studies of older people's attitudes towards technology, this demonstrates an openminded and positive attitude to some aspects of the concept. This is illustrated by the following comments drawn from the transcripts:

"People don't have time, for regular checkups on health. Sometimes getting a doctor's appointment is very difficult. If you can do the consultation through internet that would be great."

"My husband had a cardiac arrest and he didn't have any feeling it there is a pain he thinks it was just indigestion. I think this (monitoring) would have been great."

\section{Adoption}

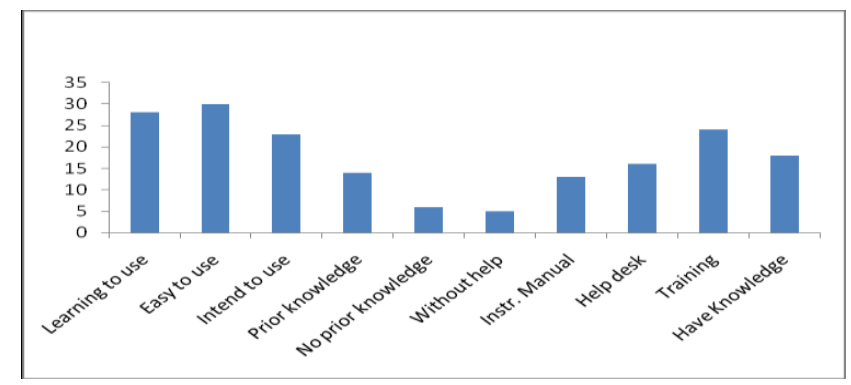

Figure 5: Factors influencing potential adoption decisions

The majority of the participants $(27$ / 34) felt that it would be easy to learn and use the technology. More than half of the participants use some sort of equipment at home for personal health monitoring. This equipment includes devices such as scales, blood pressure monitor, heart pressure and glucose., During the focus groups, participants indicated widespread use of the internet, e.g., to stay in touch with their family and friends, emails, banking, insurance and for research. Thus, participants indicated that use of the technology would not be a challenge, although a smaller proportion of participants, 22 / 34 , were definitely willing to adopt it. Participants felt that some basic prior training would be useful before use and that 
helpdesk or instruction manual might be of some help in case of problem. This is illustrated by figure 5 .

\section{Risks}

A commonly voiced risk $(23 / 34)$ related to the concern that physical contact with the physician might be lost. Moreover some of the issues highlighted during the discussion showed patients concern over the reliability of the equipment and online consultation with physician.

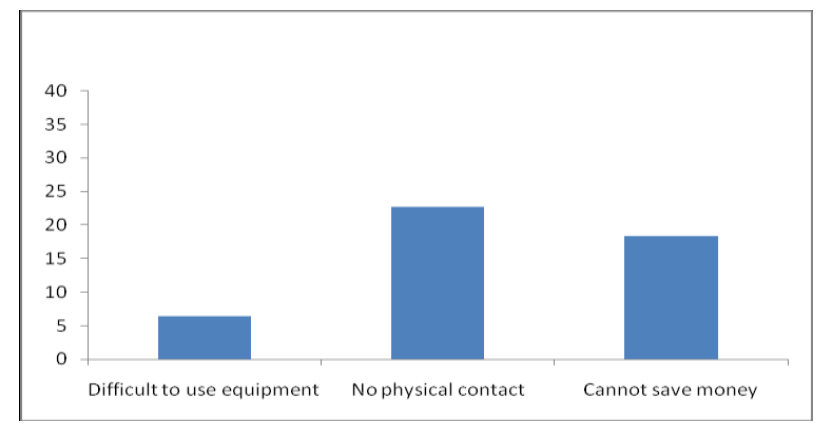

Figure 6: Concerns over potential risks

"In an emergency I would contact doctor or 999 rather than trusting the equipment."

"New technology should not interfere with existing devices like; I have a pacemaker fitted in and once my pacemaker stopped because of some equipment there."

"I will not use the equipment at all as it will make me very conscious of my health and will be wondering what is going on."

"If you go to the hospital they go through all the procedures like urine test, check weight ... you are in touch with what is going on...I am afraid you won't be getting it there."

\section{Security, Privacy and Trust}

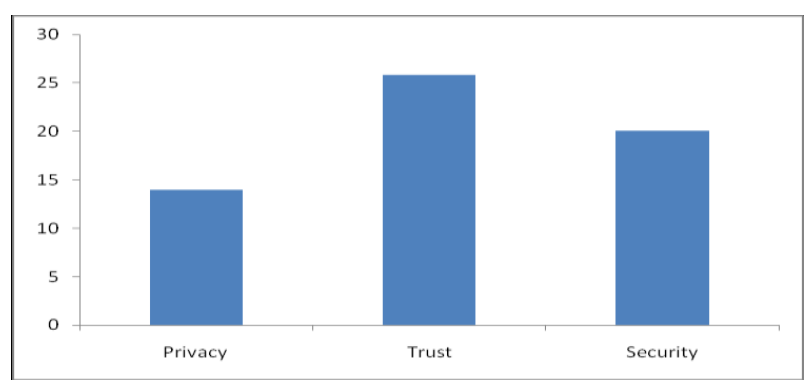

Figure 7: Attitudes towards Security, Privacy and Trust

Several participants seemed to be concerned about security (26 / 34), although, interestingly, more than half of the participants did not feel that BRAVEHALTH would violate privacy $(20 / 34)$. This was not reflected in the group discussion where participants showed great concern on privacy in data transmission to the hospital as expressed below in the following transcripts:
"I don't know what's going on in the computer and who sees my records. I would be weary of it. I am very protective."

"At the moment based on my state of health I won't be passing my information over the internet. Even if I did the hospitals prefer to use their own equipment for monitoring."

"There is a lot of problem in sending the data online as it could be accessed by anyone. If it is secure then I would use it."

"I am not sure who sees our records. I think it all depends on who is using it."

"If the health data you are passing over the internet goes to my own GP then it is fine but if goes to others then it is not good."

"If they could put security so that people can't hack then I would not worry."

\section{1) Acceptance}

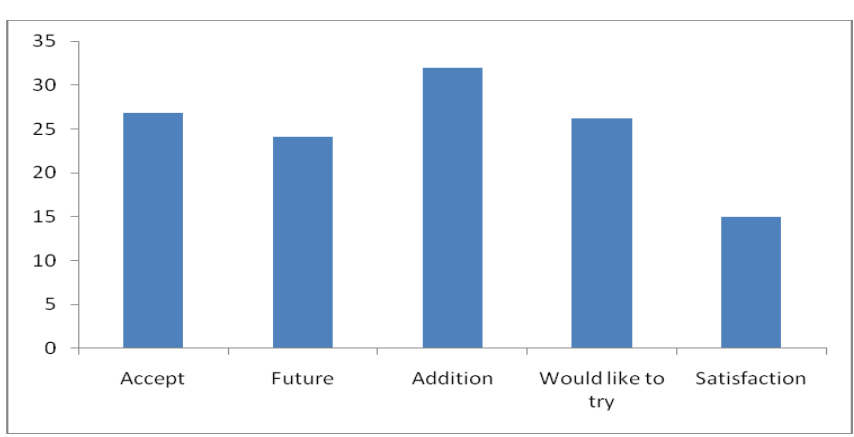

Figure 8: Factors affecting acceptance

Generally the participants (29 / 34) were willing to accept such a technology and felt it would be a great addition for their future healthcare. However, some the participants would prefer to talk to the physician rather than use video-conferencing (19 /34).

\section{2) Cell Phone}

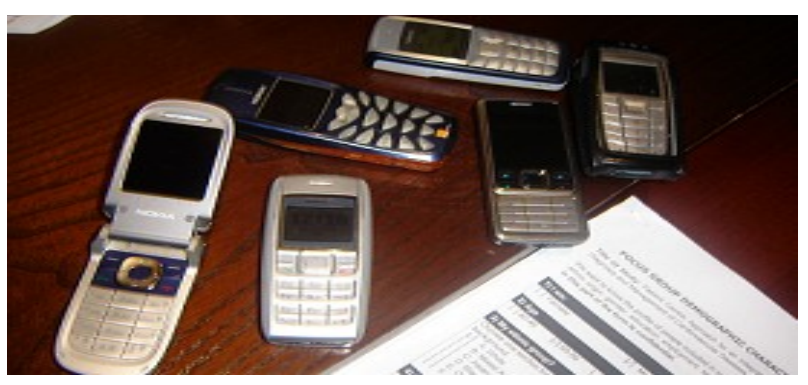

Figure 9: Patient and caregiver's mobile phone

During the focus group discussion, we found out except two, everyone has a mobile phone and most of them use their 
mobile phones for emergency or for texting, hence they seem to have their phones switched off. Also the types of phone the participants have are older phones [Figure 9] with very limited screen, as the phones seem to be passed on to them by their children.

\section{DISCUSSION}

The majority of participants felt very positive about the BRAVEHEALTH concept but (at present) not willing to adopt of the system. This could, of course, be simply due the fact that presentation of the system concept took the form of verbal descriptions rather than prototype demonstration.

Around half of the participants felt confident that they have adequate skills to handle the technology, possibly with some support through further training and access to a 'help desk'. Overall, participants in these focus groups show a positive response in regards to the potential benefits of the BRAVEHEALTH concept. In general these potential responses related to the benefits of continuous, real-time monitoring of a range of parameters related to patient condition and the capability to receive quick response to potential problems. However there remain concerns over reliability, like security, privacy and trust. These findings echo the previous studies reviewed in the introduction.

\section{ACKNOWLEDGMENT}

This study is supported by EUROPEAN UNION FP VII project number 248694 (BRAVEHEALTH).

\section{REFERENCES}

[1] Arnrich B, Mayora O, Bardram J, Tröster G. (2010) Pervasive healthcare: paving the way for a pervasive, user-centered and preventive healthcare model. Methods Inf Med.; 49(1):67-73.

[2] European Parliament Heart Group (EHN) (2009) Cardiovascular Disease Statistics. http://www.ehnheart.org/cdv-statistics.html (22 January, 2011).

[3] Lorenz B, Oppermann R. (2009) Mobile health monitoring for the elderly: Designing for diversity. Pervasive and Mobile Computing 5; 478 495

[4] Sneha S, Varshney U. (2009)Enabling ubiquitous patient monitoring: Model, decision protocols, opportunities and challenges. Decision Support Systems 46; 606-619.

[5] Varshney U. (2007) Pervasive healthcare and wireless health monitoring, Mobile Networks and Applications archive, Volume 12 , Issue 2 - 3; 113 - 127.

[6] Chan M, Estève D, Escriba C, Campo E. (2008) A review of smart homes-Present state and future challenges. Comput. Methods Prog. Biomed., Vol. 91, No. 1.; 55-81.

[7] Hung K, Zhang Y, (2003) Implementation of a WAP-based telemedicine system for patient monitoring, IEEE transactions on Information Technology in Biomedicine; 7 (2).

[8] Maglaveras N, Koutkias V, Chouvarda I, et al,. (2002) Home care delivery through the mobile telecommunications platform: the Citizen Health System (CHS) perspective International Journal of Medical Informatics 68; 99-111

[9] Jovanov E, O'Donnel A, Morgan A, et al. (2002) Prolonged telemetric monitoring of heart rate variability using wireless intelligent sensors and a mobile gateway, In Proc. Second Joint IEEE EMBS/BMES Conference.
[10] Khoor S, Nieberl K, Fugedi K, Kail E. (2001) Telemedicine ECGtelemetry with Bluetooth technology, Proceedings of Computers in Cardiology.

[11] Lymberis A, Smart A. Wearables for remote health monitoring, from prevention to rehabilitation: current R\&D, future challenges, Proc. 4th International IEEE EMBS Conference on Information Technology Applications in Biomedicine, 2003.

[12] Mendoza GG, Tran BQ, In-homewirelessmonitoring of physiological data for heart failure patients, In Proc. of the Second Joint IEEE EMBS/BMES 24th Annual Conference and the Annual FallMeeting of the Biomedical Engineering Society Conference, 2002.

[13] Lina CC, Leeb RG, Hsiaoc CC. (2008) A pervasive health monitoring service system based on ubiquitous network technology international journal of medical informatics 7 7; 461-469

[14] Sarela A, Korhonen I, Lötjönen L, et al, IST Vivago — an intelligent social and remote wellness monitoring system for the elderly, In Proceedings of the 4th Annual IEEE EMBS Special Topics Conf. on Information Technology Application in Biomedicine, 2003.

[15] Sneha S, Varshney U, Wireless ECG monitoring system for pervasive healthcare, In Proceedings of the Eleventh Americas Conference on Information Systems, AMCIS, Omaha NE, 2005.

[16] Stanford V. (2002) Using pervasive computing to deliver elder care, IEEE Pervasive Computing Magazine 11.

[17] Suzuki T, Doi M. LifeMinder: an evidence-based wearable healthcare assistant, Proc. ACM CHI Conference, 2001.

[18] Tablado A, Illarramendi A, Bermudez J, Goni A. Intelligent monitoring of elderly people, Proc. 4th Annual IEEE Conf. On Information Technology Applications in Biomedicine UK, 2003.

[19] KoivumaKi T, Ristola A, Kesti M. (2008) The perceptions towards mobile services: an empirical analysis of the role of use facilitators. Pers Ubiquit Comput; 12:67-75

[20] Steele R, et al,. (2009) Elderly persons' perception and acceptance of using wireless sensor networks to assist healthcare. International journal of medical informatics 7 8; 788-801

[21] Demiris, G., Rantz, M., Aud, M., Marek, K., Tyrer, H., Skubic, M. and Hassam, A. (2004) Older adults' attitudes towards and perceptions of 'smart home' technologies: a pilot study, Medical Informatics and Internet Medicine, 29, 87-94

[22] Katz EJ, Rice ER. (2009) Public views of mobile medical devices and services: A US national survey of consumer sentiments towards RFID healthcare technology. International journal of medical informatics 7 8;104-114.

[23] Agrell H, Dahlberg S, Jerant AF. (2000) Patients' perceptions regarding home telecare. Telemed J E Health. Winter;6(4):409-15.

[24] Haque M and Ahamed I S. (2006) Security in Pervasive Computing: Current Status and Open Issues. International Journal of Network Security, Vol.3, No.3; 203-214.

[25] Orwat C, Rashid A, Holtmann C, et al. (2010) Adopting Pervasive Computing for Routine Use in Healthcare. Pervasive Computing, IEEE, Issue 2, 64-71.

[26] Demiris G, Speedie S, Finkelstein S. (2000) A questionnaire for the assessment of patients' impressions of the risks and benefits of home telecare. J Telemed Telecare. 6(5):278-84.

[27] Jasemian Y. Elderly comfort and compliance to modern telemedicine system at home. CMPC 2008-1st International ICST Workshop on Connectivity, Mobility and Patients' Comfort.

[28] Gibbs, A. (1997). Focus Groups. Social Research Update(19).

[29] Cooper, L, and Baber, C. (2002) Focused Groups: Scenario-based discussions, in J. Langford and D. McDonagh (eds.) Focus Groups: Supporting Effective Product Development, London: CRC Press.

[30] Mitzner, T.L., Boron, J.B., Fuasset, C.B., Adams, A.E., Charness, N., Czaja, S.J., Dijksta, K., Fisk, A.D., Rogers, W.D. and Sharit, J., (2010) Older adults talk technology: technology usage and attritudes, Computers in Human Behavior, 26: 6; 1710-1721.

[31] Buckingham DC, Ahmed A, Adams EA. (2007) Using XML and XSLT for flexible elicitation of mental-health risk knowledge. Medical Informatics and the Internet in Medicine; 32(1): 65 - 81 . 
[32] Winkelman WJ, Leonard KJ, Rossos PG. (2005) Patient-perceived usefulness of online electronic medical records: employing grounded theory in the development of information and communication technologies for use by patients living with chronic illness. J Am Med Inform Assoc. May-Jun;12(3):306-14. 